\title{
EDITORIAL
}

\section{Fresh paradigms in psychotherapy for psychiatrists ${ }^{\dagger}$}

\author{
Kevin Healy
}

\begin{abstract}
Kevin Healy is Lead Clinician at the Cassel Hospital in London. Since July 2010, he has been Chair of the Faculty of Psychotherapy at the Royal College of Psychiatrists. He works clinically with young people, children, families and adults in in-patient, outreach and out-patient settings. He is particularly interested in the fresh understandings of psychotherapeutic and psychiatric practice arising from current expansion in neuroscientific knowledge. Correspondence Dr Kevin Healy, The Cassel Hospital, 1 Ham Common, Richmond, London TW10 7JF, UK. Email: Kevin.healy@ wlmht.nhs.uk
\end{abstract}

${ }^{\dagger}$ See pp. 243-249, this issue.

\begin{abstract}
SUMMARY
The recent expansion of neuroscientific knowledge is changing the nature of psychotherapeutically competent psychiatric practice. For example, components of medical psychotherapy that optimise neuroplasticity and restructure and reinforce synaptic connections can bring about enduring therapeutic changes in patients' personal functioning, experience and behaviour. By renewing their connection to the advancements of neuroscience, psychiatrists in all specialties can build their identities as professionals and their skills as practitioners, making psychotherapeutic interventions more effective for their patients.
\end{abstract}

\section{DECLARATION OF INTEREST}

None.

Chess Denman (2011, this issue) makes a strong case for training and developing psychotherapeutically competent consultant general psychiatrists who can prescribe psychotherapy rationally and assess its outcome. She suggests that, although they should be able to deliver some psychological treatments themselves, it is more important that they need to apply psychotherapeutic knowledge and emotional literacy skills to their routine clinical practice. I fully support this view. Chris Mace (my predecessor as Chair of the Royal College of Psychiatrists' Faculty of Psychotherapy) and I have recently written about the changing role of the medical psychotherapist (Mace 2011). In this commentary, I seek to convey a sense of what working in a psychotherapeutically competent manner entails, and discuss some specific shifts in psychotherapeutic thinking and practice that may make a psychotherapeutically competent approach more appealing to consultant colleagues in child and adult psychiatry.

\section{The role of the psychotherapeutically competent psychiatrist}

Psychotherapeutically competent psychiatrists will want to maintain their emphasis on providing relationships that offer opportunities for their patients to learn about and change their ways of being with themselves and others. They will recognise the advantages of working within and across multidisciplinary teams and seek to develop integrated stepped-care pathways and managed clinical networks to best coordinate therapeutic interventions. They will seek to deliver clinical services in or as near to a patient's home as possible and will welcome the increasingly influential voices of patients and their families in instigating and planning clinical interventions and service developments. All these influences on service provision push us as psychotherapeutically competent psychiatrists to be increasingly innovative and developmental in the delivery of psychologically informed treatment approaches.

Psychotherapeutically competent psychiatrists are well positioned to meet these challenges. Our role as doctors of the mind and as doctors of the body is now being more clearly defined by advances in neuroscientific knowledge of the development and functioning of the brain. This facilitates our working from an increasingly grounded and meaningful understanding of mental functioning. New and exciting findings in epigenetics and on the significance of attachment relationships are advancing our understanding of the effects of trauma, autonomic nervous system reactivity and sensorimotor bodily functioning. ${ }^{\ddagger}$ Previously emphasised differences between medical psychotherapists in theoretical understanding and in clinical practice are increasingly seen to be complementary. Combinations of differing approaches create a more whole 'body and mind' experience that better supports effective therapeutic interventions and the development of a capacity in patients to change.

\section{Fresh understandings of psychological functioning}

I would like to highlight one particular area that, to my mind, is having an increasingly significant impact on the current and future knowledge base and practice of medical psychotherapy and that is likely to be of considerable interest to psychotherapeutically competent psychiatrists. I refer to the expansion of neuroscientific knowledge on the development and functioning of the brain and of the body (Healy 2010). This now makes 
likely the development of fresh understandings of psychological functioning in health and in illness ('dis-ease') which are associated with developing meaningful psychotherapeutic, biological and social interventions. Many medical psychotherapists see this expanding knowledge base as creating an evolving sense of identity as a professional and as practitioners. They see it as a reconnection with their training as doctors of medicine. Others, some of whom are knowledgeable and some of whom are ignorant of this expanding knowledge base, are more sceptical and believe that there is nothing relevant to their work as a psychotherapeutically competent psychiatrist or medical psychotherapist in this new paradigm. Like Cozolino in the USA and Grawe in Europe, I believe that clinical practice, and consequently the lives of patients, are likely to be significantly affected by a willingness to explore and to use these new ideas.

\section{Enabling neuroplastic change}

Cozolino (2006) has highlighted components of therapy that optimise neuroplasticity and associated therapeutic changes in personal functioning. He notes that a safe and trusting relationship with an attuned, resonant, empathic therapist reactivates attachment circuitry in a patient and makes it available to neuroplastic processes. The maintenance of moderate levels of arousal maximises the biochemical processes that drive protein synthesis necessary for modifying neural structures. The activation of cognition and emotion together allows frontal systems to re-associate and re-regulate the various neural circuits that organise thinking and feeling. Cozolino suggests that the construction of a narrative that reflects a positive, optimistic self creates an evolving language for experience that can modify self-image, aid in affect regulation and serve as a guide for positive behaviour.

Cozolino suggests that differing and multiple inputs can influence the brain in complementary and additive ways. These include various combinations of significant personal relationships in therapeutic settings, encouragement to exercise regularly and to keep physically fit, engagement with an individual's spirituality, recognition of the effect of having a bond with an affectionate pet and thoughtful use of appropriate medication. Lifestyle changes, relaxation exercises, yoga and meditation can all reduce levels of arousal and maximise neuroplastic potential. Educating clients about their brains can create a common, less threatening language between client and therapist within the working relationship. Cozolino likens the optimism of the healing therapist to that of the encouraging parent. Optimism, compassion, warmth and love support the process of neuroplastic change. He concludes that the malleability of memory and the potential to rewrite history can be extremely helpful in psychotherapy.

\section{Restructuring synaptic connections}

Grawe (2006) also explores implications for psychotherapeutic practice from his reading of advances in the neurosciences. He concludes that change requires that synaptic connections that are not yet well-established are activated repeatedly, intensively and over as long a time period as possible. Working on a problem should always occur in the context of pursuing an important, currently activated, overarching goal that the patient has set. The emphasis of therapy should not be too much, or for too long, on the identification and activation of problems. Emphasis must be predominantly on altering the problem, on facilitating new thoughts, behaviour patterns, and emotions. Grawe also notes that shifting the brain towards a state that is maximally incompatible with fear results from a secure attachment relationship with a therapist who conveys competence, understanding and personal engagement.

The explicit mode of functioning is clearly better for relearning. The qualities that are linked with the explicit mode of functioning, such as conscious reflection, intention formation, planning, volitional control and verbal communication, can all be viewed as resources for therapeutic change. One can change the brain via sufficiently intensive influences in such a manner that self-sustaining new structures emerge, which then become the foundation for enduring changes in experience and behaviour.

\section{Learning together}

I believe that as medical psychotherapists we must remain open to fresh learning. In any therapeutic relationship, change is most likely to occur if it is a shared process between therapist and client. We must be prepared to continue learning and evolving as we seek to be more effective therapists. As psychotherapeutically competent psychiatrists we are professionals who pragmatically, flexibly and mindfully integrate a range of meaningful biopsychosocial interventions. We help patients, their families and carers, and their professional and social networks to address, to understand, to manage and to change severe and complex psychological and physical difficulties. We are multidisciplinary team players who offer coherence, consistency, containment and leadership qualities 
to a team. We need to be continually open to additional training to expand our knowledge, our skills and our competencies as the evidence base for what we do evolves.

In this editorial, I have suggested that the changing nature of psychotherapeutically competent practice owes much to the evolving fresh paradigm built around the recent expansion of neuroscientific knowledge. I believe that this serves to make the strong case for working in this way, presented by Chess Denman, even more appealing to child and adult psychiatrists seeking to make their range of psychotherapeutic interventions more effective for their patients. Connecting ourselves afresh to the advancements of neuroscience reconnects us to our medical training and provides a platform on which we can build our identities as professionals and our skills as practitioners.

\section{References}

Cozolino L (2006) The Neuroscience of Human Relationships: Attachment and the Developing Social Brain. W W Norton.

Denman C (2011) The place of psychotherapy in modern psychiatric practice. Advances in Psychiatric Treatment 17: 243-9.

Grawe K (2006) Neuropsychotherapy: How the Neurosciences Inform Effective Psychotherapy. Lawrence Erlbaum.

Healy K (2010) Knowledge of brain development and mental functioning opens up a fresh perspective on therapeutic interventions in psychotherapy (http://www.rcpsych.ac.uk/specialties/faculties/ psychotherapy.aspx).

Mace C, Healy K (2011) Medical psychotherapy: a specialty for now. The Psychiatrist (in press) 